2.P.H.S. Training Grant MH08034 and Research Grant MH07700 from the National Institute of Mental Health. The author acknowledges the invaluable assistance of $\mathrm{J}$. L.
Brown and R. S. Snider.

3. Present address: Psychobiology Laboratory Department of Psychology, West Virgini University, Morgantown, West Virginia 26506.

\title{
Transfer of a differentiated operant ${ }^{1}$
}

\author{
EDWARD J. RICKERT and KENNETH K \\ NISHIMOTO, Sacramento State College. \\ Sacramento, Calif. 95819
}

Twelve rats were trained to differentiate responses on a DRL schedule where the limited hold $(L H)$ of the schedule was either 2 or 4 sec. Two transfer groups (four Ss/group) were initially trained on LH4, while the remaining Ss were placed on $\mathrm{LH} 2$ condition. Following this training, transfer Ss were transferred either gradually or abruptly to the LH 2 condition. The results (1) indicate that the transfer of a differentiated operant parallels transfer of discrimination and (2) suggest that the major distinction between stimulus discrimination and response differentiation resides in the locus (external or internal) of the cue controlling behavior.

Two roles are commonly assigned response-produced (feedback) stimuli by a variety of learning theories. The earliest role ascribed feedback was to guide ensuing behavior; feedback from previous responding becomes a part of the stimulus complex to mediate subsequent behavior (e.g., Hull, 1930). The other role, more recently formulated, is to guide concurrent behavior; impending feedback may somehow mediate selection among alternative responses (e.g., Mowrer, 1960; Notterman \& Mintz, 1965). Rickert (1969) investigated these two roles by varying the temporal sequencing of response-produced stimulation in a discrete trial situation. i.e.. the relevant response-produced cue either preceded or occurred coincident with response selection. The results indicated that feedback shares a common property of external stimuli; namely, pretraining on an easy task facilitates learning a more difficult task.

The present research tests the generality of this finding by using another response dimension, namely, interresponse time (IRT) and a different (free-operant) procedure.

\section{METHOD}

Twelve naive female rats of Long-Evans stock, obtained from Simonsen Laboratories, served as Ss. The animals, ranging between 90 and 120 days of age at the beginning of experimentation, were housed separately, with water continuously available, and were maintained on a $12-\mathrm{g} /$ day ration for the duration of the study.

The experimental chamber was a conventional Gerbrands box (Model C) that measured $10 \frac{1}{2} \times 9-3 / 8 \times 10^{1 / 4}$ in. The intelligence panel housed a single paddle lever, located some 3 in. above the floor of the box, and a food receptacle, displaced to the left of the lever and situated $1 / 2$ in. above the box's floor, into which a 45 -mg Noyes rat pellet was delivered as re inforcement. Conventional electromechanical relay equipment controlled event sequences; impulse counters recorded responses.

Rats were shaped under continuous reinforcement until 50 reinforcements had been presented. Following shaping, a DRL schedule was imposed where the IRT was increased $1 \mathrm{sec} /$ day. When DRL 6 was attained, four Ss were assigned randomly to each of three groups: a gradual group (G), an abrupt group (A), and a difficult group (D). Rats in $G$ and $A$ groups were placed on the easy task: Reinforcement was contingent on selecting a response whose IRT equaled or exceeded 6 sec but was less than or equal to $10 \mathrm{sec}$. Group D was begun on the difficult differentiation; Ss were required to select IRTs that were $\geqslant$ 6 sec but $\leqslant 8 \mathrm{sec}$. That is to say. Groups $G$ and A were placed on a DRL6 LH4 schedule and a DRL6 LH2 was imposed on Group D. Ease of differentiation in this context presumably resided in the length of the IRT interval. Session length for all rats was $20 \mathrm{~min} /$ day.

After Groups $G$ and $A$ encountered eight sessions on the easy task, Group G was shifted to an immediate condition, i.e., DRL6 LH3, for two sessions. Following this training, both $G$ and $A$ were transferred to the difficult task for six sessions. Finally, to determine the effect of initial training, a subsequent test on the easy task was run for an additional six sessions with the three groups.

\section{RESULTS}

Figure 1 depicts the results in the form of percentage of correct responses for the three groups as a function of sessions. All bar-presses that terminated the IRT within the interval prescribed were recorded as correct responses. Inspection of the first eight sessions shows that performance of the groups is directly related to the length of the IRT interval. That an IRT interval of $4 \mathrm{sec}$ is indeed easier to differentiate than an interval of $2 \mathrm{sec}$ was confirmed by the analysis $[F(2,9)=10.60, p<.01]$.

Sessions 9-10 reflect performance of the groups after $G$ group was shifted to the immediate task. As may be seen, no real decrement ensued for $G$ group following the shift.

The crucial issue as to whether pretraining on an easy differentiation facilitates performance on a difficult differentiation is reflected in the set of curves from Sessions 11-16. An analysis of variance performed on the proportion of correct responses for the three groups summed over the six sessions on the difficult task indicated that significant differences exist among the groups $[F(2,9)=4.62, p<.05]$. These findings are consistent with the previous experiment that obtained transfer of differentiation with duration as the relevant response dimension (Rickert, 1969). In addition, the results conform to those observed with intradimensional, external stimulus shifts (e.g., Lawrence, 1952; Logan, 1966).

The last set of data points (Sessions 17.22) represents a shift by all groups to the easy differentiation, i.e., DRL6 LH4. Of interest here is whether or not Ss trained from the outset on the difficult differentiation would perform as well as Ss pretrained on the easy task.

An analysis performed on Sessions 17-22 for the three groups failed to reveal a difference between them, suggesting that Group D was learning about the task even though it was not reflected in their performance $[F(2,9)=1.49, p=n . s$.$] .$

\section{DISCUSSION}

Using an IRT interval as the relevant response dimension, S's performance on a difficult differentiation was facilitated by initial training on an easier differentiation. The results support the hypothesis that feedback stimuli obey the same laws as 


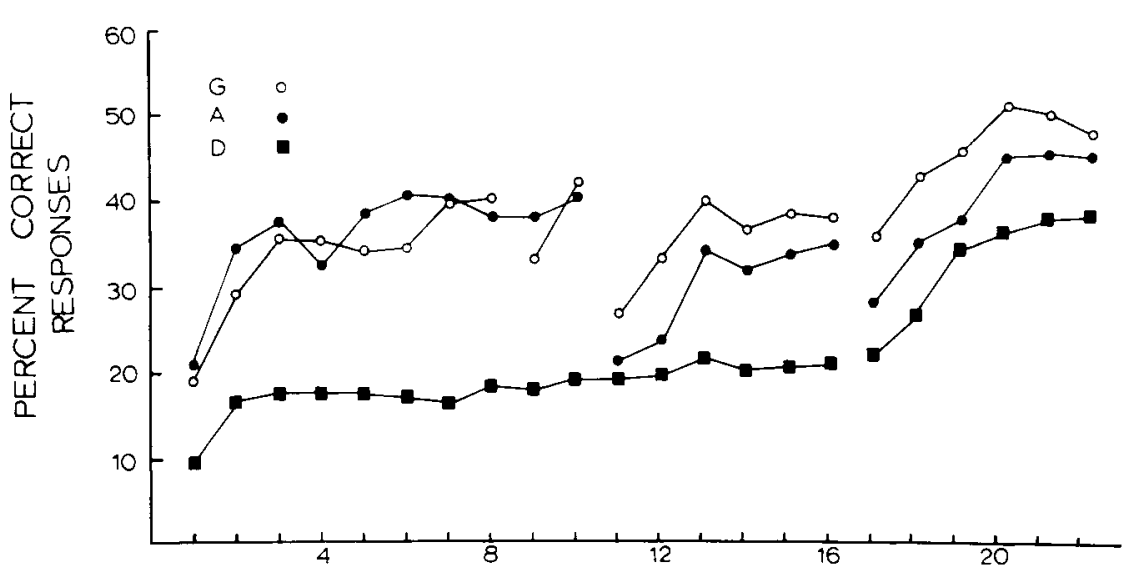

external stimuli in the sense that they facilitate intracontinuum transfer. Since the behavioral outcome of feedback is equivalent to the transfer of discrimination property of external stimuli, the results fail to support Skinner's (1938) contention that response differentiation and stimulus discrimination obey different laws.

Response differentiation is indexed as an increase in the relative frequency of some qualitative or quantitative property of behavior. Such differentiation of behavior is presumed to reside in the feedback arising from the act of emitting those
Fig. 1. Percentage of correct responses for the three groups. Breaks in the curves represent a shift by one or more groups to a different condition.

feedback is sufficient in accounting for response differentiation.

\section{REPERENCES}

HULL, C. L. Knowledge and purpose as habit mechanism. Psichological Revien. 1930. 57. $511-525$.

LAWRENCE, D. H. The transfer of a discrimination along a continuum. Journal of Comparative \& Physiological Psychology. $1952,45,511-516$.

LOGAN, F. A. Transfer of discrimination. Journal of Experimental Psychology. 1966. 71, 616-618. OMOWRER. O. H. l.caming theory and behavior. New York: Wilks 1960.

behaviors (Notterman \& Mintz, 1965). If the IRT is here treated as the elapsed time between the initiation of a response $(R n-1)$ and its ensuing member $(R n)$ and is a quantitative attribute of $R n$, then its differentiation, i.e., the selection of $R n$ rather than $\bar{R} n$, presumably resides in the feedback of $R n-1$. Thus, feedback from preceding rather than coincident behavior appears sufficient to enable choice among alternative responses. This conception of response argues against assigning two different roles to response feedback. it suggests instead that the first role ascribed

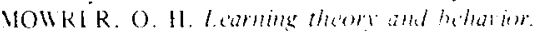
Sou rork: II iles. I4601

NOTTERMAN. J. M.. \& MIINTZ. D. I. DYnamics of response . New York: Wiley. 1965.

RICKERT. F. J. Role of leedback timuli in response discrimination and differentiation. Journal of Experimental Psychology. 1969. 82. 148-155.

SKINNER. B. I. The behavior of organisms Nin York: Appleton-Centur-Crofts. 1938. NOTES

1. This study was supported in part by the Sacramento State College Research Foundation under Grant 278.2-T. Special thanks are due Robert Essa. who assisted in the conduct of the study. 